\title{
Dinámica de Sistemas: Modelado Flexible en Logística Inversa.
}

\author{
Sandra Oviedo ${ }^{1}$, Alfredo Leiva ${ }^{1}$, Daniel Díaz ${ }^{1}$, Raymundo Forradellas ${ }^{2}$ \\ Universidad Nacional de San Juan, Facultad de Ciencias Exactas Físicas y Naturales \\ Instituto de Informática - Departamento de Informática \\ ${ }^{1}$ \{soviedo, aleiva, ddiaz\}@iinfo.unsj.edu.ar; \\ ${ }^{2}$ kike@uncu.edu.ar
}

\begin{abstract}
Resumen. Mediante la dinámica de sistemas se pueden construir modelos de sistemas complejos y realizar simulaciones ensayando diferentes escenarios, para mejorar la comprensión de los sistemas y predecir su comportamiento. Cuando se habla de flexibilidad en la industria, los autores hacen referencia a la flexibilidad de la tecnología dada por las características de la tecnología que permiten habilitar ajustes $\mathrm{u}$ otros cambios a los procesos de negocio, y a la flexibilidad del software dada por su adaptabilidad y versatilidad. Se propone la dinámica de sistemas como una herramienta flexible para el modelado. En el caso de estudio se hizo un modelo para asistir en la toma de decisiones, en dicho modelo fue posible describir flujos físicos, e inmateriales, se pudieron proponer diferentes e hipotéticos escenarios donde se puede analizar el comportamiento del sistema. Este trabajo permitió valorar la dinámica de sistemas como una metodología de modelado flexible.
\end{abstract}

Palabras claves: Software Flexible, Modelado Flexible, Dinámica de Sistemas, Logística Inversa, Herramientas para la Toma de Decisiones.

\section{Introducción}

La planificación de las actividades de reutilización, refabricación y el reciclaje de los productos también es una tarea del dominio de la logística. Es lo que se denomina logística inversa, porque el punto de partida de tales actividades no es la adquisición de materias primas, sino el usuario final [1]. Tal es el caso del empleo de los pallets en la industria, una vez usadas dichas plataformas de carga de mercaderías, son reingresadas al circuito productivo y usadas en reiteradas ocasiones.

La primer actividad de un proceso de logística inversa es la recolección de productos materiales que serán reinsertados en el proceso productivo, dado que el origen de dichos retornos son los mismos usuarios, se genera un gran problema de incertidumbre ya que hay incertidumbre acerca de las cantidades a recolectar, la localización de los materiales y productos a recolectar, el estado de los materiales y 
productos recolectados, etc.[2]. Al intentar reducir la incertidumbre mediante la comprensión del sistema se puede advertir que se trata de sistemas complejos, donde hay una gran cantidad de variables que interactúan determinando causas y efectos de manera no lineal.

Para manejar la complejidad y por lo tanto la incertidumbre, se puede emplear software que asista a la toma de decisiones. Dicho software deberá ser lo suficientemente adaptable a los permanentes cambios del medio, y versátil para poder ser usado en cualquier sistema, sea cual sea la naturaleza de la empresa que lo requiera. Ambas características, adaptabilidad y versatilidad son postuladas por [3] como condiciones que debe tener un software para ser flexible.

Se postula a la dinámica de sistemas como una herramienta de utilidad preponderante para entender sistemas complejos. Forrester introdujo el enfoque de la dinámica de sistemas a comienzos de los 60 como una metodología de modelado y simulación para análisis y toma de decisiones a largo plazo en problemas de gestión industrial dinámicos [4]. La filosofía de la dinámica de sistemas gira en torno al concepto de retroalimentación, o causalidad circular entre variables observables. Estas relaciones son, en general, suficientemente complejas de por sí. En la mayoría de los sistemas complejos existen variables observables que no están ligadas por una relación lineal causa-efecto [5].

Los sistemas complejos, lejos de caracterizarse por mostrar relaciones lineales de causalidad, suelen presentar causas y efectos que no se encuentran próximos en el espacio ni en el tiempo, y que se retroalimentan en el sistema, haciendo que su análisis mediante enfoques tradicionales resulte extremadamente complejo, tal es el caso del sistema que constituye la cadena de abastecimiento de los pallets en la industria. Tampoco es extraño en estos sistemas observar dos variables que, pese a no mantener relación alguna de causalidad, presentan alta correlación temporal simplemente como consecuencia de la dinámica global del sistema. La dinámica de sistemas es una técnica particularmente útil para analizar este tipo de sistemas caracterizados por contener complejas relaciones de causalidad circular [5].

Dada la complejidad de algunos sistemas de logística inversa y la necesidad de comprenderlos y estimar el comportamiento de los mismos, se hicieron algunos trabajos aplicando la dinámica de sistemas, la mayoría desarrollados en Europa y en Estados Unidos, (en [6] puede verse una revisión de trabajos realizados) donde las condiciones económicas, sociales y culturales son bastante diferentes a las de Argentina. Por un lado la logística inversa en la UE, se basa en la conciencia del cuidado del medio ambiente, tanto del empresariado como de la población. Por otro lado, en Estados Unidos, la principal fuerza de la logística inversa esta en la cultura de las devoluciones, cualquier consumidor sabe que puede devolver en cualquier momento de su vida útil lo que haya comprado [7].

El presente trabajo presenta un caso de estudio donde se modela una cadena de abastecimiento, de reuso y re fabricación de pallets de madera, en condiciones dadas por el contexto local, caracterizado por la extensa red de recuperadores de materiales reutilizables que existe y grandes distancias geográficas. En nuestra investigación no se encontraron trabajos similares en este contexto. 
Este trabajo tiene por objetivo destacar la flexibilidad que tiene la dinámica de sistemas en el modelado de logística inversa.

El resto de este trabajo se desarrolla de la siguiente manera, en las siguientes secciones se hace una revisión de temas como herramientas para la toma de decisiones, software flexible y modelado con dinámica de sistemas. Finalmente se presenta un caso de estudio aplicando la metodología y proponiendo algunos escenarios donde deberán tomarse decisiones.

\section{Contexto}

\subsection{Necesidad de herramientas para la toma de decisiones}

A la hora de tomar decisiones en los negocios, es necesario elegir un escenario para llevar a cabo la misma, quienes deben hacerlo, se encuentran por lo general frente a la siguiente situación: hay un conjunto de alternativas que son combinaciones de variables y es necesario decidir para lograr una serie de objetivos que se caracterizan por indicadores más o menos elevados de rendimiento. Estos objetivos abarcan conjuntos de relaciones de las empresas con diferentes partes beneficiarias con quienes interactúa en sus actividades. El objetivo de los indicadores de desempeño es medir la capacidad de las empresas para satisfacer las expectativas y necesidades de los beneficiarios, que además de ser los clientes, accionistas, asalariados, también son la comunidad y el medio ambiente, etc. También están las expectativas propias de la compañía, sobre desarrollo, crecimiento, etc. Para evaluar las alternativas de desarrollo, estas se clasifican y se elije la más pertinente. El tomador de decisiones tiene que evaluar cada alternativa, según cada uno de los criterios de selección, y luego evaluar esa selección para alcanzar los objetivos generales agregados para cada una de esas alternativas, y según el punto de vista de cada uno de los beneficiarios [8].

Resumiendo, decidir implica un proceso de selección entre varias alternativas. Una vez seleccionada una alternativa, ésta se pone en acción, con lo que indudablemente se producirán una serie de modificaciones en el sistema: los sistemas sociales son dinámicos, con lo que presentan una fisonomía distinta en cada unidad de tiempo. Esta consideración lleva a la necesidad de tomar en cuenta las consecuencias de la alternativa seleccionada, y poder evaluarlas en cuanto a sus efectos en el mediano y largo plazo.

Surge así una buena razón para justificar el uso de modelos formales: la mente humana sólo está preparada para manejar un muy reducido número de variables y en muy corto lapso de tiempo hacia el futuro.

Por tanto, con un modelo formal resultaría posible expresar concreta e indudablemente la representación del objeto, tomar en consideración todas las variables que resultan esenciales al sistema analizado, y podrían tomarse en cuenta además, los posibles efectos a mediano plazo de un cambio en alguna (o varias) de las variables consideradas cuando en los cálculos matemáticos se incluye la variable tiempo.

Para asistir a la toma de decisiones en negocios, hay tres familias de software de simulación [8]: 
- Hojas de cálculo probabilístico, como@risk, extensiones de Excel que permiten definir distribuciones probabilísticas en un conjunto de parámetros y calcular los cambios en las variables dependientes.

- Simuladores de eventos discretos como Promodel, Arena, etc., se utilizan generalmente para modelar procesos de producción de bienes o servicios, como las instalaciones de producción y centros de llamadas. Un modelo consta de las entidades, los recursos y los elementos de control (que manejan los estados de las entidades y recursos).

- Software de dinámica de sistemas, basados en la analogía de sistemas hidrodinámicos, desarrollada por J. Forrester [4], [9]. Los modelos se construyen utilizando tres tipos principales de elementos: stocks, flujos y variables auxiliares. Estos sistemas son comúnmente utilizados para la simulación de los modelos globales y el nivel estratégico. Los softwares más populares, que se utilizan en la industria como la investigación y la enseñanza son: ithink, Vensim, Stella, Powersim, GoldSim, Anyhgic, Extendi y Modelmaker (ver [10] para un análisis comparativo).

Este trabajo se enfoca en la necesidad de ayuda en el proceso de toma de decisiones a nivel gerencial o de alto nivel que tienen las empresas.

\subsection{Flexibilidad en los procesos de negocio y en el software}

La gestión de los procesos de negocio cambiantes ha sido estudiada en [11] y [12] desde tres perspectivas: estratégica, operacional, y de sistema. Los cambios en los procesos pueden ocurrir a menudo. Muchos procesos de negocio que no son bien entendidos y difíciles de formalizar, conducen a incoherencias entre las especificaciones del sistema y las necesidades reales de negocio. Además, frecuentemente existen excepciones para las rutinas básicas del negocio, requiriendo que el sistema sea robusto para gestionar estas desviaciones.

En [13] se define la flexibilidad de la tecnología como las características de la tecnología que permiten habilitar ajustes u otros cambios a los procesos de negocio. Se propone un marco de medición de la flexibilidad tecnológica que incluye factores tales como modularidad, aceptación del cambio, y consistencia en la dimensión flexibilidad estructural, tasa de respuesta, "expertise", y la coordinación de acciones en la dimensión de flexibilidad del proceso.

Zhao ha propuesto dos conceptos relacionados con la flexibilidad del software: la adaptabilidad del sistema y la versatilidad del sistema [3]. La adaptabilidad del sistema es la capacidad de modificar el sistema para hacer frente a cambios importantes en los procesos de negocios con poca o ninguna interrupción en las operaciones comerciales. La versatilidad del sistema (robustez del sistema) es la capacidad del sistema de permitir procedimientos flexibles para hacer frente a las excepciones en los procesos y procedimientos.

Más recientemente [14] abordó el tema de la flexibilidad desde las perspectivas técnicas, organizativas y humanas. Ellos clasifican la flexibilidad en cuatro 
dimensiones: la flexibilidad de los procesos, la flexibilidad inter-organizacional, la gestión y el conocimiento flexible, y la asignación de tareas flexible.

En [15] utilizan software flexibilidad integrando técnicas de workflow con técnicas de agentes inteligentes. Ellos conceptualizan software flexibilidad en dos niveles: flexibilidad tecnológica y flexibilidad del sistema. Por "flexibilidad del software", se refieren a la capacidad de una aplicación para tratar las excepciones al modelo de procesos y para enfrentar los cambios periódicos en el modelo de procesos.

Por la flexibilidad tecnológica, nos referimos a la "flexibilidad del software" que se puede lograr mediante la adopción de la combinación correcta de paradigma de modelado específico, técnica de programación, arquitectura de software y metodología de desarrollo. Este nivel de flexibilidad es análoga a la combinación de las dimensiones estructurales y de proceso propuesto en [13] pero con menos énfasis en los efectos de la organización. Por la flexibilidad del sistema, se incluye tanto a la capacidad de adaptación del sistema como a la versatilidad del sistema discutida en [3].

\subsection{Dinámica de sistemas, herramienta flexible para la toma de decisiones}

Así como las hojas de cálculo probabilístico reflejan la necesidad de tener en cuenta la incertidumbre de ciertos datos o el modelado de eventos discretos reflejan la necesidad de análisis más detallado o granularidad más fina para describir un proceso, la dinámica de sistemas responde sobre todo a la necesidad de un enfoque sistémico, en efecto, la dinámica de sistemas, integra diferentes aspectos internos y externos de la empresa, es decir relaciona la empresa con su entorno para producir un modelo global. Esto permite la toma de decisiones pertinentes y eficaces ya que se habrá podido tener en cuenta la comparación con otras organizaciones, y a la vez considerar flujos físicos e inmateriales [8]. En [9] se presenta una completa revisión de la metodología de modelado con dinámica de sistemas.

Las hipótesis de la dinámica de sistemas son las siguientes:

- Los componentes del mundo real están relacionados de manera compleja. Un sistema real puede modelarse por flujos, stocks y bucles de retroalimentación. Los flujos de información son intrínsecamente diferentes a los flujos físicos.

- La no linealidad y los delays son elementos importantes del sistema.

- El comportamiento del sistema resulta de su estructura interna, es decir, es la suma de las características de sus componentes y del conjunto de sus interacciones.

En dinámica de sistemas, el modelo empleado no solo incluirá las variables esenciales del sistema analizado o los elementos que se exteriorizan como problemáticos, sino que además permitirá considerar el sistema holísticamente como un todo orgánico y representará las interrelaciones causales que se establecen en un sistema desde la perspectiva de la teoría general de sistemas, los procesos de retroalimentación del mismo, las redes de comunicación e información internas y su contexto.

Mediante de la dinámica de sistemas se pueden construir modelos de sistemas complejos y realizar simulaciones ensayando diferentes escenarios, para mejorar la 
comprensión de los sistemas y predecir su comportamiento. La dinámica de sistemas se erige como la herramienta mediadora entre el investigador que pretende alcanzar el entendimiento de sistemas complejos, el modelado de los mismos y el soporte informático que aporta la capacidad de procesamiento.

En [2] se destaca la alta flexibilidad en la modelización.

Analizando la flexibilidad, de acuerdo a [3], de un modelo de un determinado sistema dinámico para ser empleado en la simulación durante un proceso de toma de decisiones, se puede decir que:

- Por permitir representar ambos tipos de flujos, los físicos (personas, materiales, dinero, energía, etc.) y los flujos inmateriales (información), se destaca la gran versatilidad de la herramienta.

- Mientras que las posibilidades de manejar escenarios para un mismo modelo da pautas de la gran adaptabilidad que puede tomar ante los cambios en el entorno del sistema.

\section{Caso de estudio: Modelo dinámico en la cadena de abastecimiento de una compañía de provisión de pallets para picking industrial.}

\subsection{La empresa}

"PPI" es el nombre ficticio de la empresa donde se hizo la aplicación que se presenta en este trabajo. Se trata de una PyME con base en la provincia de San Juan y que desde hace unos 10 años presta servicio en la zona oeste del país, que va desde las provincias de Salta a Neuquen y además en las provincias de Córdoba y eventualmente en Buenos Aires. Cuenta con una nómina de 15 empleados.

El servicio que brinda PPI consiste en el alquiler de pallets para transporte y almacenamiento de mercadería. Los pallets que utiliza son de madera por lo tanto entre sus principales actividades esta la de refabricación de pallets, es decir que cuando están deteriorados los pallets son reparados, en algunos casos se recuperan pallets completos y en otros, se rescatan las partes que servirán en otros pallets. El servicio incluye el transporte de pallets desde y hacia las empresas demandantes. Actualmente se manejan con una existencia de alrededor de 15.000 unidades.

PPI se provee de pallets de distinta fuente, pueden comprar pallets usados o nuevos (se compran por lote y cuando son usados, no todos vienen en buenas condiciones, por lo cual será necesario hacer recuperaciones), o fabricar pallets nuevos, eso dependerá de las condiciones del mercado.

Satisfacer en tiempo y forma a los clientes es el gran desafío que tiene PPI, lo cual se convierte en un complejo problema, muy dinámico, ya que es fundamental coordinar el transporte que rescatará pallets de un cliente para llevar a otros, preferentemente dicho transporte será un camión vacío que coincida en su ruta en el momento que están los pallets disponibles para ser retirados o entregados. Ese transporte puede ser propio o de un tercero contratado oportunamente. 
Los clientes manejan estacionalidad en los pedidos, habiendo algunas épocas de demanda pico coincidiendo con los tiempos de producción y entrega en la industria vitivinícola, principalmente.

\subsection{Objetivo}

Se espera modelar el sistema que involucra el abastecimiento de pallets para las actividades de la empresa, a fin de entender el sistema complejo y lograr mediante la simulación, dar respuestas a preguntas tales como: “cuántos pallets será necesario adquirir para un determinado período de tiempo?" , "cuántos pallets se estima que pedirán los clientes para el mes próximo?”, “cuánta es la deficiencia permitida en el transporte?", "será necesario contratar transportes por anticipado?", "qué pasa si la cantidad de empleados destinados la recuperación de pallets es de tantos?”,etc.

\subsection{Metodología de trabajo}

La metodología de trabajo empleada, coincide con lo que Valerie Gacogne llama "Etapas de la construcción del modelo de simulación" [16] consta de 4 etapas:

- Definición del problema, objetivos, ámbito de aplicación del modelo.

- Diseño del modelo del sistema: el modelo verbal y su representación en forma de diagrama de influencias (o diagramas de causalidad circular).

- Formalización del modelo matemático para la transcripción de los diagramas de influencias en las ecuaciones utilizando el software de modelado (variables de calibración).

- Las simulaciones del modelo: prueba de hipótesis de base de análisis dinámico respuestas de las pruebas de sensibilidad de los modelos, comentarios sobre la construcción del modelo y correcciones (incluyendo la percepción de la realidad que ha sido modificado por una mejor comprensión de cómo funciona el sistema), la validación de modelos y definición de escenarios.

En el presente trabajo se empleó la versión trial 9.0.2 de STELLA, un producto de Isee Systems, STELLA es un lenguaje de programación orientada a objetos que utiliza una interfaz gráfica para facilitar la construcción de estructuras de sistemas dinámicos, una completa guía de uso se ofrece en [17].

\subsection{El modelo}

\section{Diagrama de influencias.}

Una vez acordado con el interlocutor de la empresa PPI se establecieron los objetivos y límites del modelo y se realizó conjuntamente el diagrama de influencias, Figura 1. Lo cual brindó una primera aproximación al modelo. 


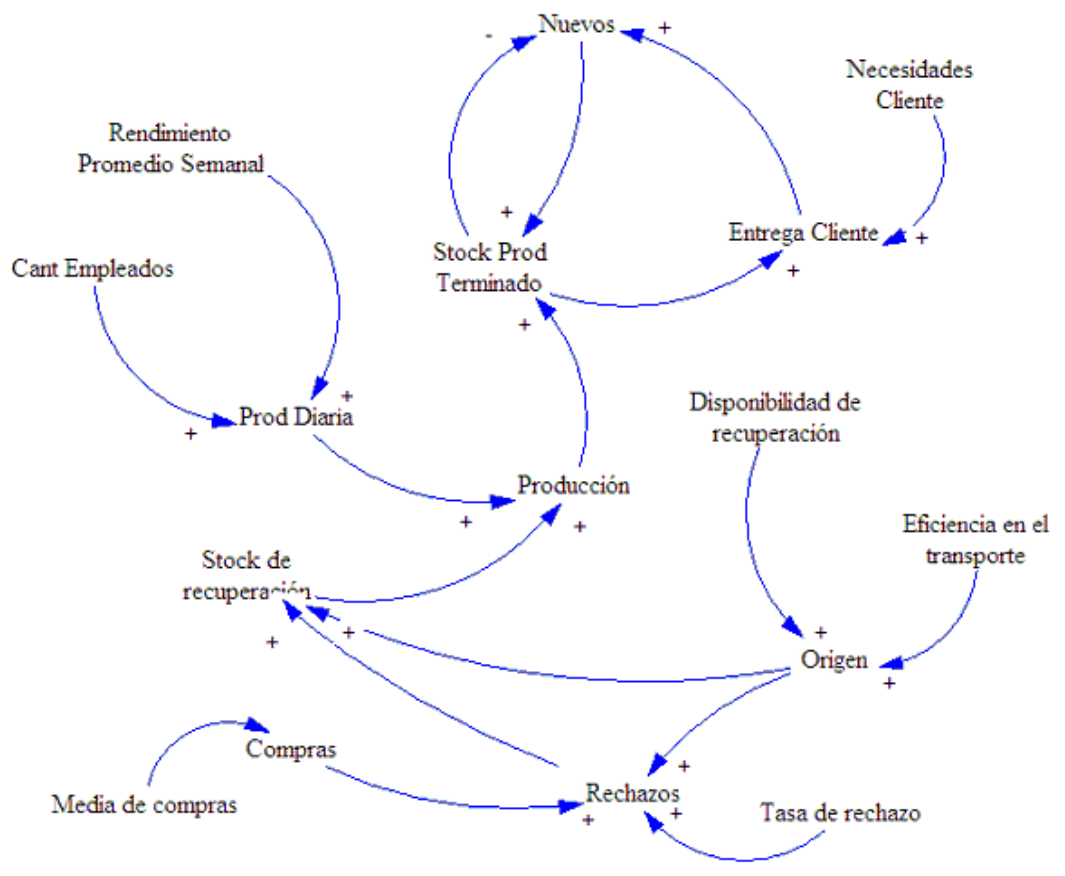

Figura 1. Diagrama de influencias

Se destaca la utilidad de los diagramas de influencias para la comunicación de ideas como facilitadores de la comunicación entre quien modela y el experto conocedor del sistema que se modela, lo cual permitió una mejor comunicación y las oportunas correcciones sugeridas por el conocedor del sistema.

\section{Diagrama de Forrester.}

Luego se procede la obtención del diagrama de Forrester, a fin de lograr el modelo formal, Figura 2. Este diagrama se obtuvo en base al diagrama de influencias, se agregaron algunos parámetros a fin de dar más adaptabilidad al sistema, pero se mantuvieron las mismas variables y sus relaciones causales.

Como se dijo, a las variables ya definidas, se agregaron algunas otras variables que son usadas para introducir parámetros necesarios para estimar algunas variables que responden a ciertas distribuciones estadísticas, como el caso de "Necesidades Cliente", "Disponibilidad de Recuperación", entre otras.

Una vez realizado el diagrama de Forrester, se establecen las relaciones cuantitativas entre las diferentes variables alcanzándose el modelado formal. 


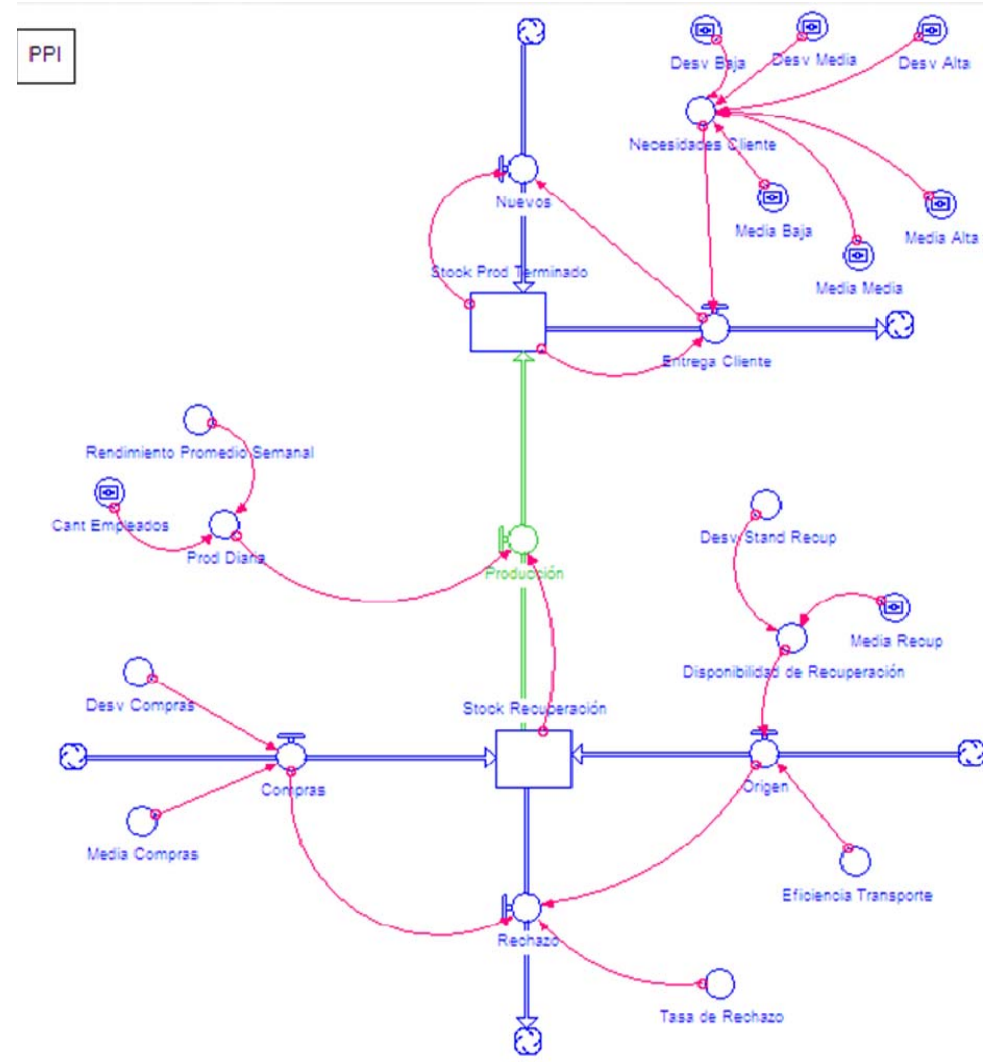

Figura 2. Diagrama de Forrester

\section{La simulación.}

Una vez realizado el modelo formal, se hacen las verificaciones y validaciones necesarias a fin de asegurar en lo posible la calidad del modelo, a continuación se plantean los escenarios de interés para analizar el comportamiento del sistema.

La Figura 3 muestra el resultado de una simulación, la flexibilidad del software permite elegir qué variables ver en la simulación. Se pueden emplear los Slider Input Devices para introducir valores de variables independientes del sistema, de esa manera se pueden determinar los escenarios para las diferentes simulaciones.

De acuerdo al modelo planteado, en este caso algunos escenarios posibles son:

- Surge un nuevo competidor en la región, lo cual provocaría una disminución en la demanda y en el ingreso de pallets usados. Cambiando los parámetros de demanda y aprovisionamiento se podría evaluar los requerimientos de transporte, mano de obra para recuperación, de compras, para plantear futuras estrategias. 
- Aumento de costos en la mano de obra, se puede evaluar los requerimientos mínimos de mano de obra, analizando el aprovisionamiento de pallets y la evolución del stock de pallets para recuperación.

- Demoras en las devoluciones por parte de los clientes, quienes toman decisiones en PPI podrán evaluar hasta cuánto se puede esperar a que los clientes hagan las devoluciones, con sólo modificar el parámetro de "tasa de recuperación", sin presionar a los clientes a que devuelvan los pallets antes de que sean necesarios, lo cual podría ocurrir en caso de incertidumbre.

Como puede observarse, a partir del mismo modelo se pueden analizar distintas circunstancias que propone el mercado día a día y cambios en el contexto de la empresa.
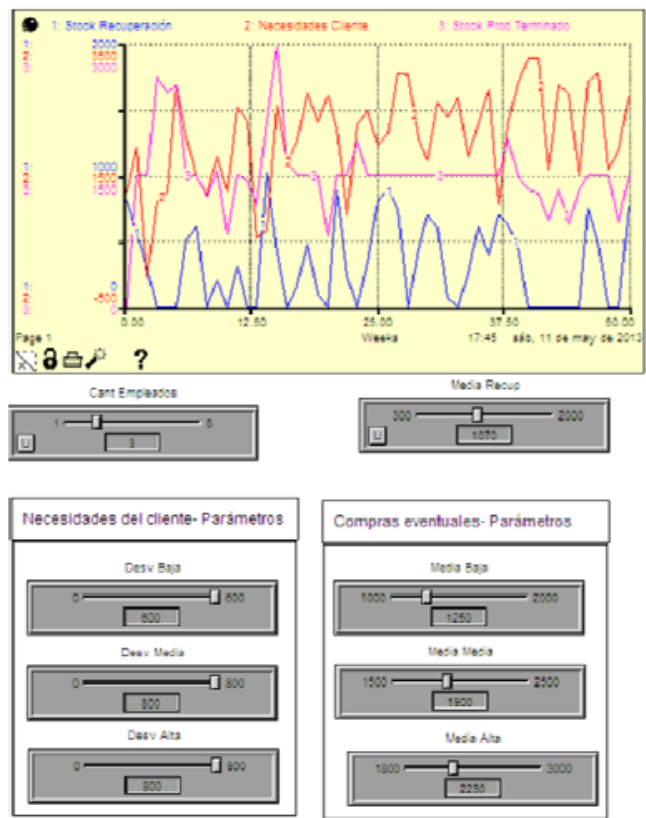

Figura 3. Resultado de una simulación. Slider input devices que facilitan la definición de diferentes escenarios.

\section{Conclusiones y futuros trabajos}

El presente trabajo destaca la necesidad del uso de herramientas software para ayuda en la toma de decisiones. Analizando, en particular, la complejidad de los sistemas de logística inversa, se propone la dinámica de sistemas como metodología y su soporte de software para atender dicho problema.

Dada la dinámica que presenta el contexto de las actividades de la industria y en particular, de la logística inversa, se pone en evidencia la necesidad de que dichas 
herramientas de software sean lo suficientemente adaptables a los cambios de contexto y circunstancias a que pueda estar sometida la organización. También se requiere que el software empleado sea lo suficientemente versátil para modelar los flujos que definen la estructura interna del sistema, dichos flujos serán de distinta naturaleza, físicos e inmateriales. Habiendo identificado estas condiciones se puede hablar de la gran flexibilidad de la dinámica de sistemas.

En el caso de estudio se pudo demostrar que con dinámica de sistemas se pudo lograr un modelo que describe el ámbito de toma de decisiones, en dicho modelo fue posible decribir flujos físicos, y no físicos. Y se pudieron proponer diferentes e hipotéticos escenarios donde se puede analizar el comportamiento de las variables para tomar decisiones.

En este sentido se puede decir que la dinámica de sistemas provee:

- Por un lado flexibilidad tecnológica, definida como el conjunto de características de la tecnología que permiten habilitar ajustes u otros cambios a los procesos de negocio.

- Y por otro lado, flexibilidad del software que considera la adaptabilidad del sistema como la capacidad de modificar el sistema para hacer frente a cambios importantes en los procesos de negocios con poca o ninguna interrupción en las operaciones comerciales, y la versatilidad del sistema (robustez del sistema) como la capacidad del sistema de permitir procedimientos flexibles para hacer frente a las excepciones en los procesos y procedimientos.

Como futuros trabajos se propone:

- Respecto al análisis de flexibilidad. Se podrían establecer parámetros de evaluación más formales, que podrían adecuarse al marco de medición de la flexibilidad tecnológica que incluye factores tales como modularidad, consistencia en la dimensión flexibilidad estructural y/o tasa de respuesta, propuesto por [13].

- En cuanto al modelo en PPI, por completitud para el análisis del sistema, podrían agregarse las variables de costeo.

\section{Referencias}

1. M.P. de Brito, and R. Dekker, "Reverse Logistics - a framework" Erasmus University-Holanda 2003.

2. S. Rubio Lacoba, "El Sistema de Logística Inversa en la Empresa: Análisis y Aplicaciones. Tesis Doctoral," Departamento de economía aplicada y organización de empresas, Universidad de Extremadura- España.2003.

3. J.L. Zhao, "Intelligent Agents for Flexible Workflow Systems," Conference on Information Systems, Baltimore. Maryland.1998.

4. J. Forrester, "Industrial Dynamics.," MIT Press, Cambridge.1961.

5. L. Izquierdo, and J. Galan, " Modelado de sistemas complejos mediante simulación basada en agentes y mediante dinámica de sistemas," EMPIRIA. Revista de Metodología de Ciencias Sociales2008. 
6. P. Georgiadis, and D. Vlachos, "Desition Making in Reverse Logistics Using System Dynamics," Yugoslav Journal of Operations Research2004, pp. 259272.

7. S. Oviedo, and R. Forradellas, "Una propuesta de Logística Inversa. Caso de estudio: Aplicación en Manufactura,” Tesis de maestría2010.

8. Y. Bernard, and M. Camargo, "La conception industrielle des produits. Volume 2," Lavoisier - Hermes Science Publications 2008.

9. S. Oviedo, M. Camargo, and P.C. Narváez Rincón, "Contribution de la Dynamique des Systèmes à la modélisation de scenarii pour l'evaluation de la filière de production de biodiesel," Tesis Master Science, Technologie et Santè- ENSGSI-2010.

10. M. Elhamdi, and B. Yannou, "Evaluation des flux de valeurs d'une entreprise: une approche de dynamique des systémes multicritére," CIGI'056éme Congrés International de Génie Industriel2005.

11. M.J. Earl, "The new and the old of business process redesign," Journal of Strategic Info. Systems1994, pp. 5-22.

12. C. Ellis, and G. Rozenberg, "Dynamic change within workflow systems. ," COOCS 95, Milpitas, CA.1995.

13. K.M. Nelson, H.J. Nelson, and M. Ghods, "Technology flexibility: conceptualization, validation, and measurement". ," HICSS971997.

14. W. Deiters, T. Goesmann, and T. Löffeler, "Flexibility in Workflow Management: Dimensions and Solutions," International Journal of Computer Systems Science and Engineering2000, pp. 303-313.

15. D.D. Zeng, and J.L. Zhao, "Achieving Software Flexibility via Intelligent Workflow Techniques," 35th International Conference on System Sciences2002.

16. V. Gacogne, "Les modèles de simulation systémiques, outils d'aide à la décision?," Atelier pratique $d u$ territoire et de la complexitéComplexio.2008.

17. TEAM, "Technical Documentation for the iThink \& STELLA Software.," isee systems, inc. 2007. 